\title{
Optimal DG Siting and Sizing in Distribution System using PSO-DE Approach
}

\author{
Damanjeet kaur \\ Assistant Professor, UIET, Panjab University, Chandigarh
}

\begin{abstract}
In this paper a population based approach for optimal sizing and sizing of distributed generation (DG) is implemented. The proposed approach is based on Particle Swarm Optimization algorithm (PSO) and Differential Evolution (DE) in combination. As distribution system operates at LV so losses are more. To overcome this problem, DG placement is carried out. DG site and size is important for better performance of the system. Keeping this in view, the problem of DG sizing and siting is solved in a such a manner that cost of power losses is minimized while constraints of voltage is within the limits. The proposed PSO-DV approach has advantage of sufficient randomness and gives near optimal solution with less computation burden. The proposed approach is tested on 33-node test system. The results obtained using proposed approach is compared with heuristic based approach. On comparison it is found that the results obtained using suggested approach is better than existing method.
\end{abstract}

\section{Introduction}

Distribution system is the final link between transmission network and consumers. Distribution system has wide variety of consumers and shares major portion of power losses. The load demand on distribution system always keeps on changing and this growth is a continuous phenomena which results in voltage drop and more losses. But for distribution system the major constraint is to maintain voltage profile. To maintain voltage profile or minimize losses, various techniques are carried out. One way is to expand/upgrade the existing system while other is to postpone the construction of new one by adding capacitors or inclusion of distributed generation (DG). As per IEEE, distributed generation is defined as the generation of electricity by facilities that are sufficient smaller than central power plants and can be connected at nearly any point in power system. DG may give various benefits as voltage control power quality, loss reduction, system reliability etc. DG can be effective in power loss minimization or voltage profile improvement if size and site of placement is properly selected otherwise these DG may have adverse affects. DG has advantage of supplying active and reactive power in power system. For last one decade the researchers are working continuously for optimal siting and sizing of DG is carried out.

For last decade, evolutionary techniques became very common because of their applicability to various types of variables and diversity in population. For last one decade the researchers are working continuously for optimal siting and sizing of DG is carried out. Authors devised various techniques analytical, artificial Bee colony for

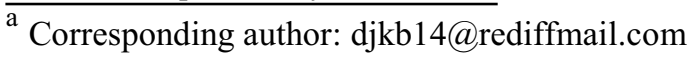

DG siting and sizing [1-5]. These days authors have solved the problem of DG siting and sizing and shunt capacitor simultaneously [6-9] in order to reduce power losses. Among these techniques, PSO has become common because of its simplicity and maintaining balance between local minima and global minima which helps to find solution of the problem in particular direction. Local minima of individual and global minima of a group are used to generate new population. It is observed that sometimes PSO traps in local minima and converges very quickly in few iterations. To overcome this problem, there is need to increase diversity in population. In this paper, to increase diversity, other population based approach differential evolution is also combined. Hence in this paper, a population based approach for Distributed generation siting and sizing is implemented which gives optimal solution in less computational time.

\section{Problem Formulation}

\subsubsection{Objective Function}

In DG siting and sizing problem, the aim is to select DG in such a manner that the cost of power losses is minimized while voltage constraint is satisfied i.e.

$\operatorname{Min} \mathrm{Z}=\sum_{f \in F D} I_{f}^{2} R_{f}$ 
Where $I_{f}$ - Current flowing through a branch $f$

$R_{f}$ - Resistance of branch $f$

$F D$-Total number of feeder segments in a feeder

\subsubsection{Constraints}

\section{(i) Voltage Constraint}

The size of DG should be selected such that voltage at each node $n p$ should be within the prescribed limit as:

$$
V_{\min }<V_{n p}<V_{\max }
$$

\section{(ii) Power Balance Constraint}

The total active power generated by generators and DGs should meet load demand and power losses i.e.

$$
\sum_{g=1}^{N g} P g_{g w / D G}+\sum_{G=1}^{N D G} P g_{G}=P_{d}+P_{L}
$$

Where

$N_{g^{-}}$Number of traditional generators in given system

$N D G$-Number of DG's

$P g_{g w / D G}-$ Operating active power of traditional power generation unit $\mathrm{g}$ with introduction of $\mathrm{DG}$

$P_{d^{-}}$Total load demand

$P_{L-}$ Total active power losses

$P g_{G}$-Active power of DG unit $G$

\section{Assumptions for DG Sizing and Siting}

(i) DG is working at upf.

(ii) DG is supplying only real power.

(iii) Only single DG can be placed.

\section{Solution Approach}

The proposed approach consists of PSO and DE in combination to increase the search diversity in order to get optimal solution for the same. In the proposed approach PSO explores the potential optimal regions within reasonable short period of time and then DE searches this space rapidly. Therefore the proposed approach is able to find global solution of the problem.

The algorithm of the proposed approach is as described below [10-12]:

\section{A. Generation of Population}

Initially a population of individuals is generated. In PSO, individuals are termed as particle and group of population is known as swarm. Each particle $p$ at $k^{\text {th }}$ iteration has velocity $\left(\mathrm{Vel}_{u}^{k}\right)$ and position $\left(\operatorname{pos}_{u}^{k}\right)$ within the search space. In this paper, each particle represents the discrete sizes of conductors for $n b r$ feeder segments.

\section{B. Fitness Function}

To find optimal size of conductor, fitness function is defined corresponding to the objective function. For each particle $p$, fitness function is evaluated.

\section{Find Local Best and Global Best}

After calculation of fitness function, the best fitness function for each particle and among swarm is found out. The best fitness function of particle $p$ is known as Pbest and that of swarm is defined as Gbest.

\section{At First Iteration}

After calculation of fitness, at iteration $u=1$, Gbest is considered equal to Pbest. Compare the fitness value of the particle $p$ at $u+1$ with that of the previous best one.

\section{E. Choose Value of Probability}

A value of probability variable $(\mathrm{P})$ is chosen in order to apply either PSO or DE for next generation. The value is set by the user.

\section{F. Updation of Velocity and Position Vector}

If value of $\mathrm{P}$ is less than the user defined value then update velocity and position vector of particle $p$ using Gbest and pbest till iteration $u+1$ using following equations.

$$
\begin{gathered}
V e l_{p}^{u+1}=\left(\text { wVel }_{p}^{u}+c_{1} \operatorname{rand}_{1}\left(\text { pbest }- \text { Pos }_{p}^{u}\right)+\right. \\
c_{2} \operatorname{rand}_{2}\left(\text { gbest }- \text { Pos }_{p}^{u}\right) \\
\operatorname{pos}_{p}^{u+1}=\operatorname{pos}_{p}^{u}+\operatorname{Vel}_{p}^{u+1}
\end{gathered}
$$

where rand 1 and rand 2 are random numbers generated in $[0,1]$; $\mathrm{c} 1$ an $\mathrm{c} 2$ are acceleration constant; $w$ is the inertia weight factor, it provides balance between global and local explorations. $w$ often decreases from 0.9 to 0.4 during the iterations. It is generally set using the following equation:

$$
w=w_{\max }-\left(\left(w_{\max }-w_{\min }\right) / k_{\max }\right) * k
$$

where $U_{\max }$ is the maximum number of iterations and $u$ is the current number of iteration.

Otherwise apply mutation, crossover and selection operators of DE as given in the following sections.

\section{G. Mutation}

To produce population for next iteration, $u=u+1$, a mutation operator is applied at the current population at $\mathrm{u}=1$. Three particles $\left(P_{1, u}, P_{1, u}, P_{1, u}\right)$ are selected randomly and a mutant vector is generated as given below: 


$$
M u \tan t=P_{3, u}+F\left(P_{1, u}-P_{2, u}\right)
$$

where $F$ is constant having value between 0 and 1 .

H. Crossover

To increase diversity in search space, in DE trial vectors are generated by applying crossover between earlier generation at $u^{\text {th }}$ iteration and mutant vector. The trial vector is obtained by comparing the random number generated between 0 and 1 and crossover probability selecting a crossover probability $\left(\mathrm{P}_{\text {cross }}\right)$. If random number is greater than the $P_{\text {cross }}$ then design variable of trail vector will be from mutant vector otherwise from $u^{\text {th }}$ iteration generation.

\section{Selection}

The selection is carried out if the trial vectors will be part of new population or not. To decide this, fitness function of trail vector is calculated and compared with Pbest. If it is better than earlier then Pbest, then trial vector is considered as particle for new generation and store new value of Pbest.

\section{J. Updation of Pbest and Gbest}

Update Pbest and Gbest corresponding to best trial vector.

\section{K. Stopping Criteria}

The above steps of subsection $E$ to $J$ is repeated till the search satisfies the termination condition. The termination condition may be maximum number of iterations or the convergence criteria set.

\subsection{Algorithm for Distributed Generation}

(i). Choose values $c_{1}, c_{2}$, number of particles and number of iterations.

(ii). Perform sensitivity analysis of real power losses w.r.t active power injection as given in [13].

(iii). Sort nodes in descending order corresponding to their sensitivity obtained.

(iv). Select first $n$ number of nodes as candidate locations for DG placement.

(v). Generate a group $(n)$ of particles for candidate locations within the $d$-dimensional search space of the problem i.e. the size of distributed generation alongwith position and velocity of each particle.

(vi). Modify population as in Section 3.

(vii). Repeat above Steps till maximum number of iterations are achieved.

\section{Results and Discussions}

The proposed approach is tested on $12.66 \mathrm{kV}, 33$ bus radial distribution system is considered. The total real and reactive load is $3.72 \mathrm{MW}$ and 2.3 MVAR. The line and load data is given in [5].

Initially sensitivity analysis of real power losses w.r.t active power injection for the given data is carried out. The nodes are placed in descending order corresponding to the sensitivity value as given in Table 1 . First of all 15 nodes are considered as candidate nodes for DG siting. A set of population is generated for given number of candidate nodes. Fitness function for the generated population is calculated. Store Pbest and Gbest value of the current iteration of population. Update these values position and velocity values as explained in Section 3.

Table 1-Nodes in Descending order corresponding to sensitivities

\begin{tabular}{|c|c|}
\hline $\begin{array}{l}\text { Nodes in Descending order } \\
\text { corresponding to sensitivity }\end{array}$ & Value of Sensitivity \\
\hline 18 & -0.0013 \\
\hline 17 & -0.0013 \\
\hline 16 & -0.0012 \\
\hline 15 & -0.0012 \\
\hline 14 & -0.0012 \\
\hline 13 & -0.0012 \\
\hline 33 & -0.0011 \\
\hline 32 & -0.0011 \\
\hline 31 & -0.0011 \\
\hline 12 & -0.0011 \\
\hline 11 & -0.001 \\
\hline 30 & -0.001 \\
\hline 10 & -0.001 \\
\hline 29 & -0.001 \\
\hline 9 & -0.0009 \\
\hline 28 & -0.0009 \\
\hline 8 & -0.0008 \\
\hline 27 & -0.0008 \\
\hline 7 & -0.0007 \\
\hline 26 & -0.0007 \\
\hline 6 & -0.0007 \\
\hline 5 & -0.0005 \\
\hline 25 & -0.0005 \\
\hline 24 & -0.0004 \\
\hline 4 & -0.0004 \\
\hline 23 & -0.0003 \\
\hline 3 & -0.0003 \\
\hline 22 & -0.0001 \\
\hline 21 & -0.0001 \\
\hline 20 & -0.0001 \\
\hline 19 & -0.0001 \\
\hline 2 & 0 \\
\hline
\end{tabular}

After a given number of iterations, the best solution obtained is as mentioned in Table 2. It is found that considering DG of size $50 \%$ of total loading results 1.4567 MW DG placement at bus 12 which causes power losses of 122.69 MW. DG placement causes minimum voltage at bus 33 of 0.9379 p.u. On comaprison with existing heuristic approach it is clear from Table 2 that the proposed approach yields better results than existing 
method. The suggested approach has less power losses as compared to other method.

Table 2-Comparison with Other Method for 50\% Loading

\begin{tabular}{|c|c|c|c|c|}
\hline & $\begin{array}{l}\text { Total } \\
\text { Real } \\
\text { Losses } \\
\text { (MW) }\end{array}$ & $\begin{array}{l}\text { \%Loss } \\
\text { Reduction }\end{array}$ & $\begin{array}{l}\text { Optimal } \\
\text { Location }\end{array}$ & $\begin{array}{l}\text { Minimum } \\
\text { Voltage in } \\
\text { the System } \\
\text { (p.u.) }\end{array}$ \\
\hline Base Case & 213.3 & - & - & $\begin{array}{l}0.9065 \text { at } \\
\text { Bus } 18\end{array}$ \\
\hline $\begin{array}{l}\text { Heuristic } \\
\text { Approach }\end{array}$ & 142.34 & 22.83 & $\begin{array}{l}1.0 \text { MW } \\
\text { DG at bus } \\
18\end{array}$ & $\begin{array}{l}0.9311 \text { at } \\
\text { bus } 33\end{array}$ \\
\hline $\begin{array}{l}\text { Proposed } \\
\text { Approach }\end{array}$ & 122.69 & 42.48 & $\begin{array}{l}1.4567 \\
\text { MW DG at } \\
\text { bus } 12\end{array}$ & $\begin{array}{l}0.9379 \text { at } \\
\text { bus 33 }\end{array}$ \\
\hline
\end{tabular}

The results are also compared for size of DG as 50\% of total loading with heuristic approach in which only node number 17 and 18 are considered as sensitive nodes. On implementation it is found that The suggested approach yields better results than existing heuristic approach as tabulated in Table 3 .

Table 3-Comparison of results with same search space for $50 \%$ loading

\begin{tabular}{|c|c|c|c|c|}
\hline & $\begin{array}{l}\text { Total Real } \\
\text { Losses } \\
(\mathrm{MW})\end{array}$ & $\begin{array}{l}\text { \%Loss } \\
\text { Reduction }\end{array}$ & $\begin{array}{l}\text { Optimal } \\
\text { Location }\end{array}$ & $\begin{array}{l}\text { Minimum } \\
\text { Voltage in } \\
\text { the System } \\
\text { (p.u.) }\end{array}$ \\
\hline $\begin{array}{l}\text { Base Case } \\
\text { (No DG) }\end{array}$ & 213.3 & - & - & $\begin{array}{l}0.9065 \text { at } \\
\text { Bus } 18\end{array}$ \\
\hline $\begin{array}{l}\text { Heuristic } \\
\text { Approach }\end{array}$ & 142.34 & 22.83 & $\begin{array}{l}1.0 \text { MW } \\
\text { DG at bus } \\
18\end{array}$ & $\begin{array}{l}0.9311 \text { at } \\
\text { bus } 33\end{array}$ \\
\hline $\begin{array}{l}\text { Proposed } \\
\text { Approach }\end{array}$ & 138.1950 & 35.21 & $\begin{array}{l}0.9886 \\
\text { MW DG } \\
\text { at bus } 17\end{array}$ & $\begin{array}{l}0.9310 \\
\text { bus } 33\end{array}$ \\
\hline
\end{tabular}

\section{Conclusions}

In this paper a population based approach for DG placement in radial distribution system is proposed. The problem of DG siting is solved using sensitivity analysis method of real power losses w.r.t active power injection which gives candidate nodes for DG siting. For fixed number of candidate nodes, DG sizing problem is solved using particle swarm optimization and DE approach in combination. The results obtained are compared with heuristic approach. On comparing the results it is found that proposed approach is suitable to find quality solution in less computational time.

\section{References}

1. F.S Abu-Mouti and M.E.El-Hawary, "Optimal distributed generation allocation and sizing in distribution systems via artificial bee colony algorithm," IEEE Transactions on Power Delivery, No 26 Vol. 4, pp 2090-2101, 2011.

2. M. R. Haghifam, H. Falaghi and O.P. Malik, "Riskbased distributed generation placement. IET Generation, Transmission and Distribution, Vol. 2, pp252-60, 2008.

3. N. Acharya, P Mahat, and N. Mithulananthan, “An analytical approach for DG allocation in primary distribution network.," Electric Power Systems Research, Vol. 28, No.10, pp 669-678, 2006.

4. P. S. Georgilakis and N. D. Hatziargyriou, "Optimal distributed generation placement in power distribution networks: Models, methods, and future research," IEEE Transaction on Power Systems Vol. 28, pp 3420-8, 2013.

5. T.N Shukla, S.P. Singh, V. Srinivasarao, and K.B. Naik, "Optimal sizing of distributed generation placed on radial distribution systems," Electrical Power Component System, Vol. 38, No. 3, pp 260274, 2010.

6. S.A. Taher, M. Hasani and A. Karimian, "A novel method for optimal capacitor placement and sizing in distribution systems with nonlinear loads and DG using GA," Communications in Nonlinear Science and Numerical Simulation, Vol. 16, No 2, pp 851862, 2011.

7. S.M Sajjadi, M.R. Haghifam, and J. Salehi, "Simultaneous placement of distributed generation and capacitors in distribution networks considering voltage stability index," International Journal of Electrical Power \& Energy Systems, No. 46,pp 366375, 2013.

8. V. A. Evangelopoulos and P.S. Georgilakis, "Optimal distributed generation placement under uncertainties based on point estimate method embedded genetic algorithm," IET Generation Transmission and Distribution, Vol 8, pp 389-400, 2014.

9. S. Gopiya Naik, D.K. Khatod, M.P.Sharma, " Optimal Allocation of Combined DG and Capacitor for Real Power Loss Minimization in Distribution Networks," Electrical Power and Energy Systems, Vol 53, pp 967-973, 2013.

10. J. Kennedy and R. Eberhart, "Particle Swarm Optimization," IEEE Proceedings on 1st International Conference on Neural Networks (Perth, Australia), 1942-1948, 1995.

11. J. Kennedy and R. Eberhart, "A Discrete Binary Version of the Particle Swarm Algorithm," IEEE Conference on Systems, Man, and Cybernetics, Orlando, FA, 4104-4109, 1997.

12. K. Price, R. Storn and J. Lampinen, Differential Evolution-A Practical Approach to Global Optimization, Springer, Berlin Heidelberg New York, 2005.

13. D. K. Khatod, V. Pant, and J.D. Sharma, "A Novel Approach for Sensitivity Calculations in the Radial 
Distribution System," IEEE Transactions on Power Delivery, vol. 21, no. 4, pp. 2048-2057, October 2006. 